\title{
GENERATION OF THE 30 M-MESH GLOBAL DIGITAL SURFACE MODEL BY ALOS PRISM
}

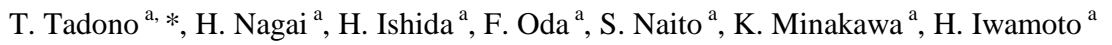 \\ ${ }^{a}$ Japan Aerospace Exploration Agency (JAXA), 2-1-1, Tsukuba, Ibaraki, 305-8505 Japan \\ - (tadono.takeo, nagai.hiroto, ishida.haruyuki, oda.fumiko, naito.shino, minakawa.kenta, iwamoto.hiroyuki)@jaxa.jp
}

Commission IV, WG IV/3

KEY WORDS: Global, High resolution, DEM/DTM, Satellite, Mapping

\begin{abstract}
:
Topographical information is fundamental to many geo-spatial related information and applications on Earth. Remote sensing satellites have the advantage in such fields because they are capable of global observation and repeatedly. Several satellite-based digital elevation datasets were provided to examine global terrains with medium resolutions e.g. the Shuttle Radar Topography Mission (SRTM), the global digital elevation model by the Advanced Spaceborne Thermal Emission and Reflection Radiometer (ASTER GDEM). A new global digital surface model (DSM) dataset using the archived data of the Panchromatic Remote-sensing Instrument for Stereo Mapping (PRISM) onboard the Advanced Land Observing Satellite (ALOS, nicknamed "Daichi”) has been completed on March 2016 by Japan Aerospace Exploration Agency (JAXA) collaborating with NTT DATA Corp. and Remote Sensing Technology Center, Japan. This project is called "ALOS World 3D" (AW3D), and its dataset consists of the global DSM dataset with 0.15 arcsec. pixel spacing (approx. $5 \mathrm{~m}$ mesh) and ortho-rectified PRISM image with $2.5 \mathrm{~m}$ resolution. JAXA is also processing the global DSM with 1 arcsec. spacing (approx. $30 \mathrm{~m}$ mesh) based on the AW3D DSM dataset, and partially releasing it free of charge, which calls "ALOS World 3D 30 m mesh" (AW3D30). The global AW3D30 dataset will be released on May 2016.

This paper describes the processing status, a preliminary validation result of the AW3D30 DSM dataset, and its public release status. As a summary of the preliminary validation of AW3D30 DSM, $4.40 \mathrm{~m}$ (RMSE) of the height accuracy of the dataset was confirmed using 5,121 independent check points distributed in the world.
\end{abstract}

\section{INTRODUCTION}

Topographical information is fundamental to many geo-spatial information and applications on the Earth. Earth observation satellites have an advantage in collecting such information because they are capable of repeated global observation.

In the past, several satellite derivative datasets were provided to examine global terrains with medium resolutions. The Shuttle Radar Topography Mission (SRTM), which is spearheaded by the National Aeronautics and Space Administration (NASA) and the National Geospatial-Intelligence Agency (NGA) of the United States (Farr et al., 2007), has operated in 2000 and generated digital elevation model (DEM) with originally 3 arcsec. spacing (approx. $90 \mathrm{~m}$ ) covered latitude regions within +/-60 degrees. On September 2014, the White House was announced that the enhanced global SRTM data is to be released with 1 arcsec. spacing (approx. $30 \mathrm{~m}$ ) in 2015 (NASA JPL, 2014).

Another dataset is the global DEM (GDEM), which used data acquired by the Advanced Space-borne Thermal Emission and Reflection Radiometer (ASTER GDEM), was released in 2009 by NASA and the Ministry of Economy, Trade and Industry (METI), Japan, and a second version was released in 2011 (Tachikawa et al., 2011). This dataset has 1 arcsec. spacing as its specification, but problems with its quality still remain. SRTM version 3 was produced by combining SRTM and GDEM, and was released in 2012.
A new global digital surface model (DSM) dataset using the data acquired by the Panchromatic Remote-sensing Instrument for Stereo Mapping (PRISM) onboard the Advanced Land Observing Satellite (ALOS, nicknamed "Daichi") was launched by the Japan Aerospace Exploration Agency (JAXA) in collaboration with commercial partners NTT DATA Corp. and Remote Sensing Technology Centre of Japan (RESTEC) in 2013. This project is named "ALOS World 3D" (AW3D), and the dataset created consists of fine resolution DSM ( 0.15 arcsec., approx. $5 \mathrm{~m}$ ) and ortho-rectified image (ORI, approx. $2.5 \mathrm{~m}$ resolution) of PRISM in global terrestrial area within $+/-80 \mathrm{deg}$. latitude regions. It was used approx. 3 million scenes of PRISM acquired from 2006 to 2011 that corresponding to ALOS's mission life. AW3D version 1 was completed on March 2016, and it is distributing by NTT DATA and RESTEC in commercial bases.

JAXA is also processing the global DSM with 1 arcsec. spacing based on the AW3D DSM dataset, which calls "ALOS World 3D $30 \mathrm{~m}$ mesh" (AW3D30), and partially releasing it free of charge on April 2016 to contribute not only research and science communities but also commercial uses in fields of geospatial information and application (JAXA EORC, 2016). The complete global dataset will be released on May 2016 as version 1. In this paper, we describe the processing status, a preliminary validation result of the AW3D30 DSM dataset, and its public release status. The existing global DEM and worldwide distributed independent check points were used for a preliminary validation of AW3D30 DSM.

\footnotetext{
* Corresponding author.
} 

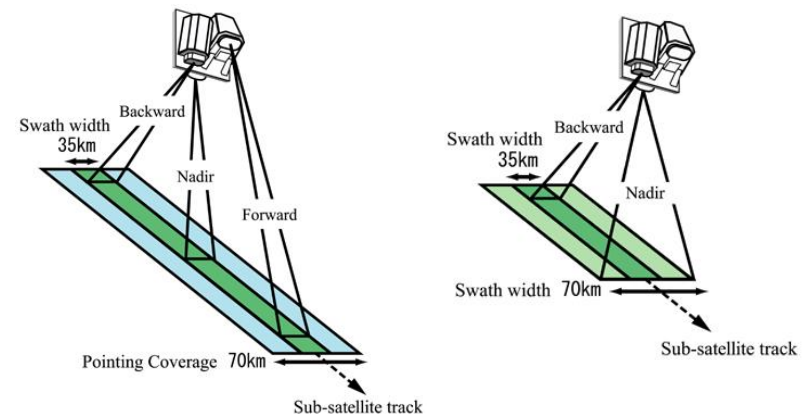

Figure 1. Observation geometries of PRISM triplet observing mode (OB1, left), and stereo (by nadir plus backward) observing mode (OB2, right).
Table 1. PRISM characteristics.

\begin{tabular}{lc}
\hline \hline \multicolumn{1}{c}{ Item } & Description \\
\hline Number of bands & 1 (Panchromatic) \\
Wavelength & $0.52-0.77$ micrometers \\
Number of optics & 3 (NDR, FWD, and BWD) \\
Base to height ratio & 1.0 (between FWD and BWD) \\
Spatial resolution & $2.5 \mathrm{~m}$ (NDR) \\
Swath width & $35 \mathrm{~km}(\mathrm{OB} 1) / 70 \mathrm{~km}(\mathrm{OB} 2)$ \\
Signal to noise ratio & $>70$ \\
MTF & $>0.2$ \\
Pointing angle & $-1.2 /+1.2$ degrees (OB1, NDR) \\
Bit length & 8 bits/pixel \\
Data rate & 960 Mbps (OB1, OB2) \\
Data compression & Lossy, JPEG extension (onboard) \\
Data downlink rate & $240 \mathrm{Mbps}(1 / 4.5$ compression) \\
\hline \hline
\end{tabular}

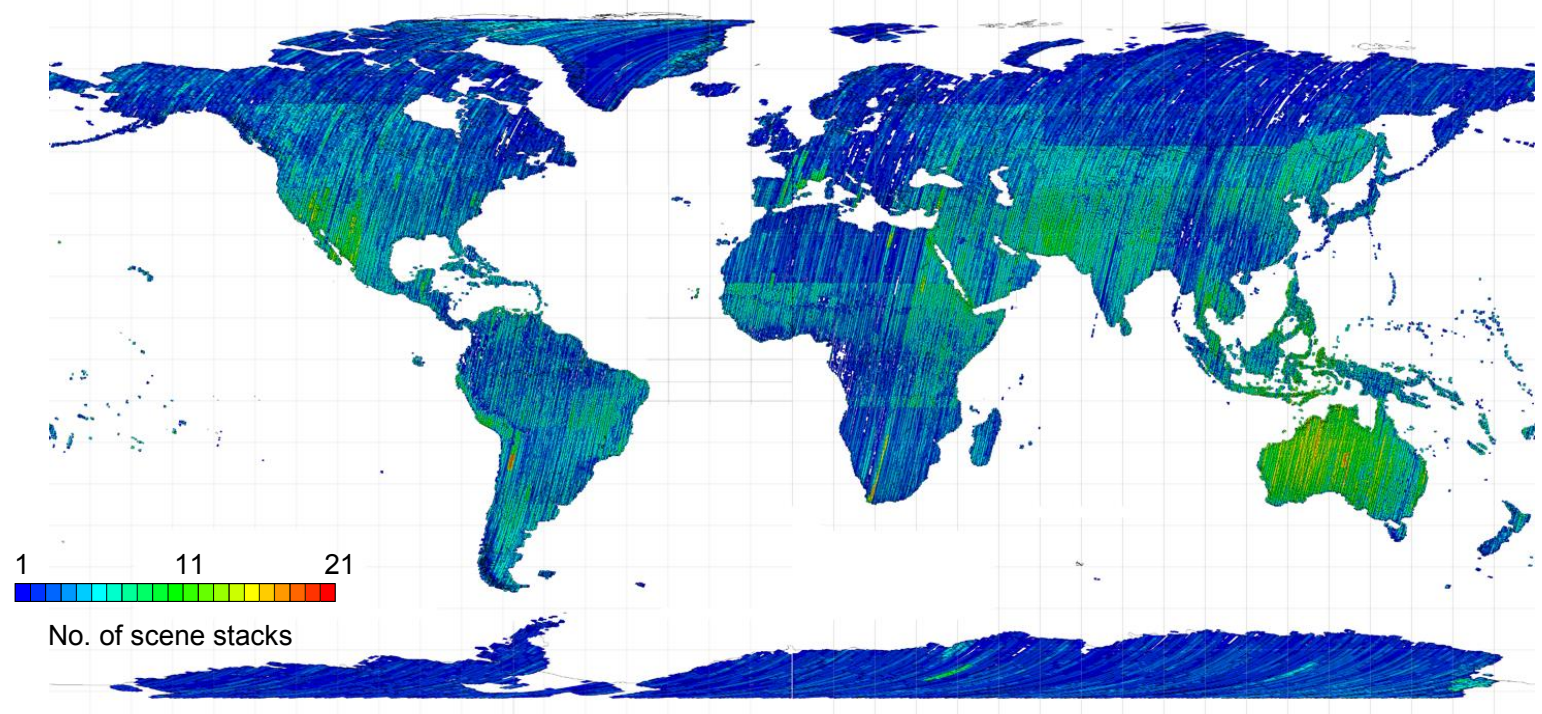

Figure 2. The status of PRISM stereo scenes in the archive with $<30 \%$ cloud cover.

Table 2. Specifications of the "ALOS World 3D" (AW3D) dataset.

\begin{tabular}{|c|c|}
\hline Product Name & Specifications / Descriptions \\
\hline 1) Precise DSM Dataset & 1 deg. tile of lat/long frame unit \\
\hline DSM file (DSM) & $\begin{array}{l}\text { The geoid height of GRS80 ellipsoid model with ITRF97 coordination ( } \mathrm{m} \text { unit). } \\
\text { Equivalent lat/long, Signed } 16 \mathrm{bit} \text { raster data, GeoTIFF format } \\
\text { Horizontal spacing: } 0.15 \text { arcsec (approx. } 5 \mathrm{~m} \text { ) } \\
\text { Accuracies: } 5 \mathrm{~m} \text { in height (RMSE), } 5 \mathrm{~m} \text { in geolocation (RMSE) }\end{array}$ \\
\hline Mask file (MSK) & $\begin{array}{l}\text { Masked out due to clouds, land water, and ocean in individual tile. } \\
\text { 8bit raster data, GeoTIFF format }\end{array}$ \\
\hline Stack number file (STK) & $\begin{array}{l}\text { Stacking number of the scene-frame DSM for individual pixel. } \\
\text { 8bit raster data, GeoTIFF format }\end{array}$ \\
\hline Header information (HDR) & Processing information for generating the dataset in ASCII text \\
\hline Scene list (LST) & Scene information of source scene-frame DSM in ASCII text \\
\hline $\begin{array}{l}\text { Quality assessment } \\
\text { information (QAI) }\end{array}$ & $\begin{array}{l}\text { Summary of the quality assessment information (e.g. stats of differences with } \\
\text { existing DEMs etc.) in ASCII text }\end{array}$ \\
\hline 2) ORI Dataset & Nadir image, Scene-frame unit \\
\hline Ortho rectified image (ORI) & $\begin{array}{l}\text { ITRF97 and GRS80 coordination, GeoTIFF format. } \\
\text { Horizontal spacing: } 0.075 \text { arcsec (approx. } 2.5 \mathrm{~m} \text { ) } \\
\text { Geolocation accuracy: } 5 \mathrm{~m} \text { (RMSE) }\end{array}$ \\
\hline Header file (OHR) & Source data information in ASCII text \\
\hline $\begin{array}{l}\text { 3) Correlation Coefficient Image } \\
\text { (CCI) }\end{array}$ & 8bit raster data, GeoTIFF format (Intermediate product) \\
\hline
\end{tabular}

\section{ALOS AND PRISM}

ALOS was launched on Jan. 24, 2006, and operated very well throughout the mission target life of five years (Shimada et al., 2010), however its mission operation has been terminated on May 12, 2011 due to a power generation anomaly. Although, approx. 6.5 million scenes of global archived data are still available for users.
PRISM was an optical instrument onboard ALOS that consisted of three radiometers for nadir- (NDR), forward- (FWD), and backward- (BWD) looking, and had a $2.5 \mathrm{~m}$ spatial resolution with a $35 \mathrm{~km}$ swath width in triplet observing mode (OB1). It also had the capability to obtain NDR and BWD images in stereo observing mode (OB2) (Tadono et al., 2009). Figure 1 illustrates the PRISM observation geometries, and Table 1 


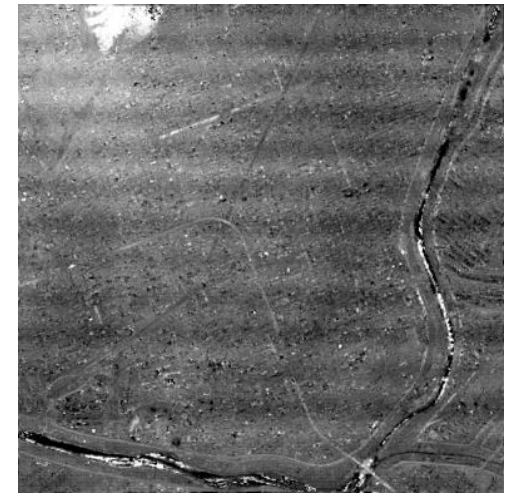

(a) DSM processing with PAD.

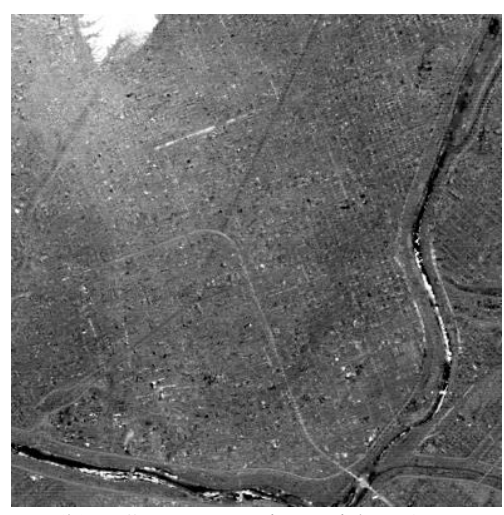

(b) DSM processing with HAD.

Figure 3. Comparison between the PRISM DSM processed with (a) PAD and (b) HAD (Takaku and Tadono, 2010).

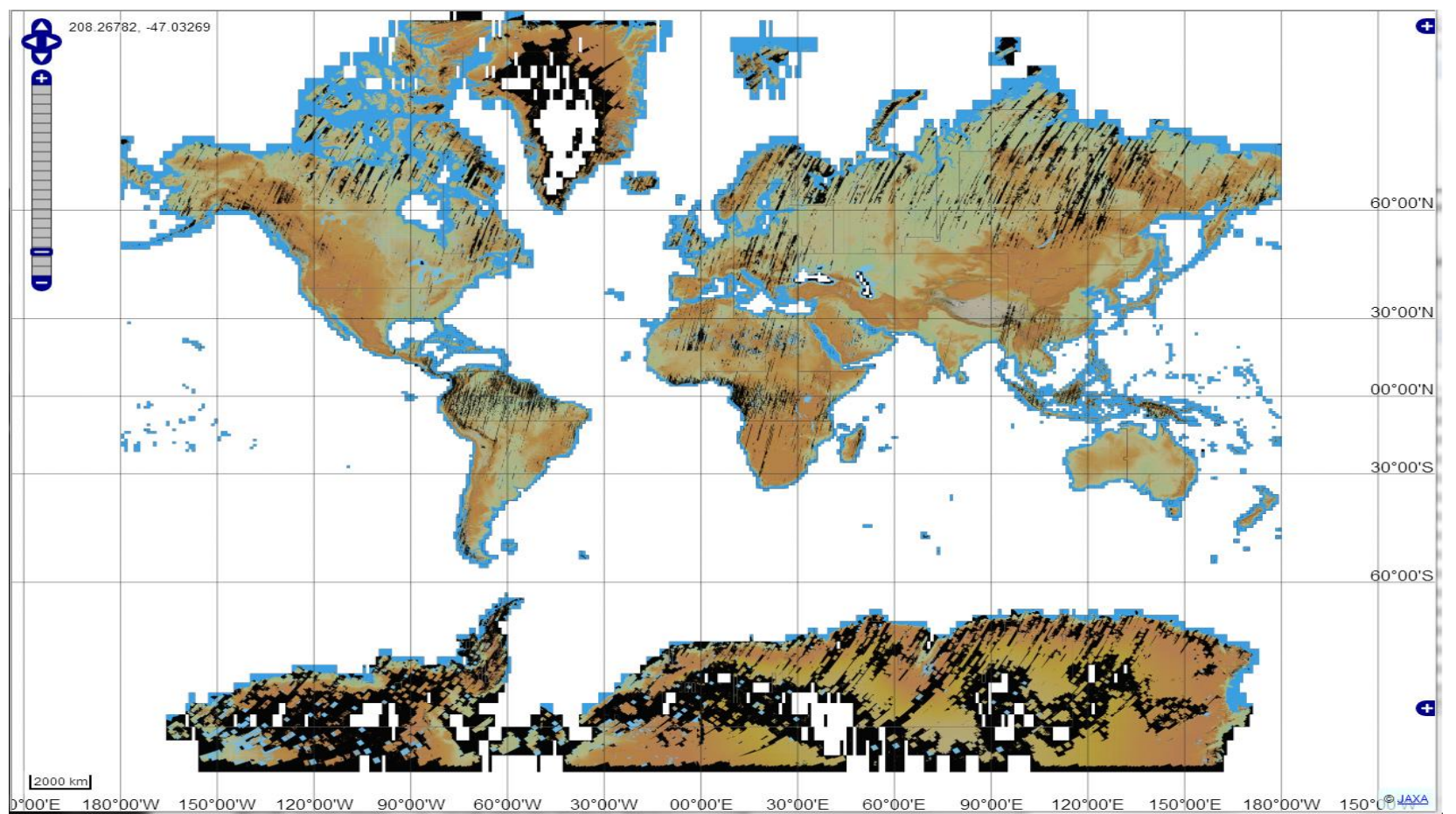

Figure 4. The browse image of AW3D global DSM data (as of March 2016).

summarizes the primary PRISM characteristics. Their images perform along-track stereo observation to generate DSM. Figure 2 shows a map of PRISM stereo scenes in the archives with less than $30 \%$ cloud cover in a scene that is approx. 3 million scenes. The AW3D dataset was basically produced using these scenes; therefore, a processing system with a huge capacity for both level 1 and DSM/ORI had to be used.

\section{DATASET GENERATION}

\subsection{Specifications of the AW3D dataset}

Table 2 summarizes the specifications of the AW3D dataset. It consists of the DSM dataset in 1 degree tiles of lat/long frame units, and ORI dataset of NDR in scene bases. As mentioned earlier, the DSM has 0.15 arcsec. horizontal spacing (approx. 5 $\mathrm{m}$ ) with $5 \mathrm{~m}$ height accuracy in root mean square error (RMSE) as the target accuracy. This is achieved by stack processing using multi-temporal acquired scenes of DSM to reduce uncertainty, cloud covers, and noises. The pixel-bases stacking number is indicated in the stack number file (STK), and the remaining clouds, water areas, ocean, and low correlation such as snow and ice areas are indicated in the mask file (MSK). The processing information, the quality assessment information (QAI), and scene ID list used to create a DSM tile are also contained in the dataset, respectively. In the case of AW3D30 DSM dataset, similar files and information are composed. AW3D30's QAI file is added the corresponding information to AW3D's one, which is just for reference.

The ORI is processed for NDR only due to necessity and limitation of data storage. It has 0.075 arcsec. horizontal spacing, which corresponds to approx. $2.5 \mathrm{~m}$ original resolution. The correlation coefficient image (CCI) is an intermediate product of the image matching processing in DSM generation. A summary of CCI is given in QAI file.

\subsection{Level 1B1 processing}

The input data for processing PRISM DSM/ORI software is level $1 \mathrm{~B} 1$ of the standard processing product, however a problem was reported from a previous study (Takaku and Tadono, 2010) that the attitude fluctuations of the satellite sometimes cause jitter noises to appear in the generated PRISM 
DSM. Operationally, the attitude data of PRISM is provided at $10 \mathrm{~Hz}$ of sampling rate called the precision attitude data (PAD). It is composited the data of the star tracker (STT) and the inertial reference unit (IRU) (Iwata et al., 2008). Meanwhile, the high-frequency attitude data (HAD) is produced at a sampling rate of $675 \mathrm{~Hz}$ by compositing PAD and the angler displacement sensor (ADS) for the on-orbit technical validation purposes. Figure 3 shows an image comparison example of DSM processed using PAD and HAD. Jitter noises can be seen in along-track direction in the DSM processed with PAD, however such noises do not appear in the DSM processed with HAD. HAD cannot completely remove all jitter noises in DSM, but it does greatly reduce them as shown in Fig. 3. Therefore, a huge amount of level 1B1 with HAD processing system was developed in the JAXA Earth Observation Research Center to process input data for the global PRISM DSM/ORI dataset.

\subsection{AW3D dataset generation}

We are developing software called the "DSM and Ortho-image Generation Software for ALOS PRISM (DOGS-AP)" to process the scene- and manual-bases DSM and ORI using PRISM stereo image pairs (Takaku and Tadono, 2009a). DOGS-AP also has an instrument calibration function. Since the launch of ALOS, we have carried out the calibration of PRISM to improve the absolute accuracy of the standard products (Tadono et al., 2009, 2010). This result is also reflected in the DOGS-AP software. As a result, GCPs are not essential in processing PRISM DSM and ORI because its standard product has good planimetric accuracy of $6.1 \mathrm{~m}$ (RMSE) for nadir radiometer.

In past several years, the generated PRISM DSMs were also validated at several test sites with different surface conditions (Takaku and Tadono, 2009b, 2010). The confirmed height accuracies of PRISM DSMs fell in the range 2.94 to $7.15 \mathrm{~m}$ (RMSE), $\sim 3.44 \mathrm{~m}$ (bias) and $\sim 6.44 \mathrm{~m}$ (standard deviation, STDEV). In addition, in a previous study we also investigated the generation of a DSM mosaic to cover large-scale areas and concluded that the bias error correction only has to consider the height, even if the GCPs were not used in processing them (Takaku and Tadono, 2011).

The automatic processing algorithm had to consider expanding the area globally, which is considered based on the experiences of previous studies. The detail of the algorithm is given by Takaku et al. 2016.

Figure 3 shows the browse image of AW3D global DSM data that indicates the processing status as of end of March 2016. In total, 23,521 tiles have been completed to cover approx. +/- 80 degrees latitude regions. The black colours represented MSK file due to remaining clouds, land water areas, and snow and ice areas. This is covered Greenland and Antarctica but there are many masked areas due to snow and ice. Also, around equator and high latitude areas are remaining clouds that are corresponding to availability of PRISM data shown in Fig. 2.

\subsection{AW3D30 dataset generation}

AW3D30 DSM dataset is generating based on the AW3D DSM dataset, which is applied two resampling methods to obtain one pixel value on AW3D30 from 7 by 7 pixels on AW3D. The first one is used the averaging method (Ave), which is simplify calculated an average value from appropriate 49 pixels except for masked out value. This is likely to reduce the fraction of masked pixels as a merit, however the spatial resolution may be

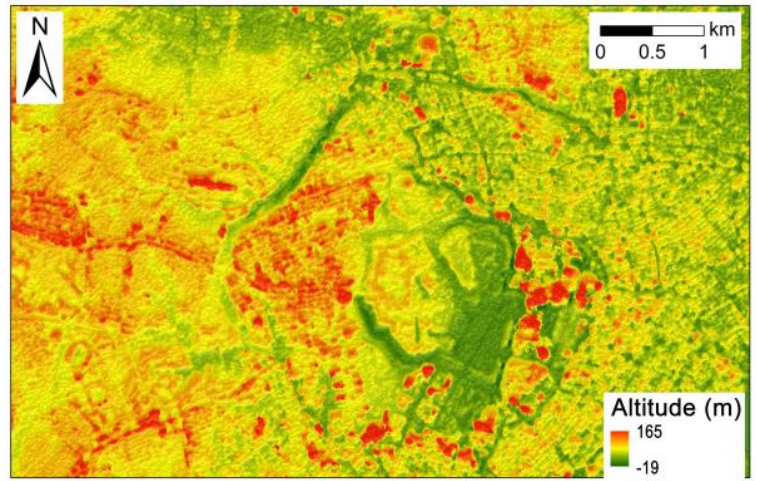

(a) AW3D DSM (5 m mesh).

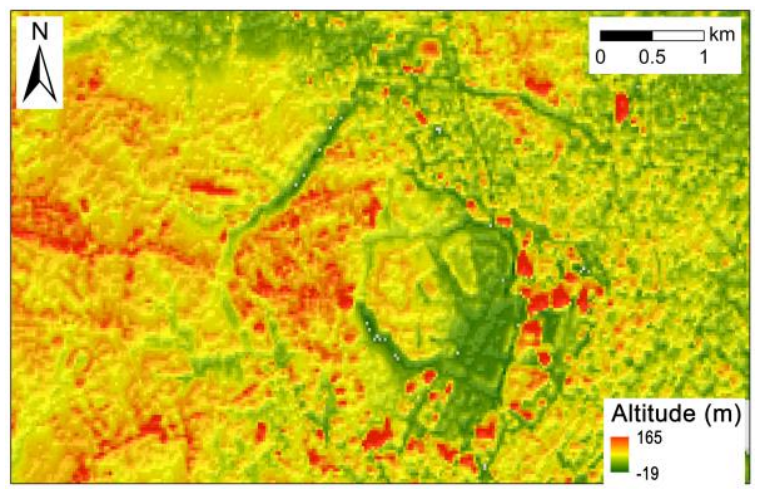

(b) AW3D30 Ave DSM (30 m mesh).

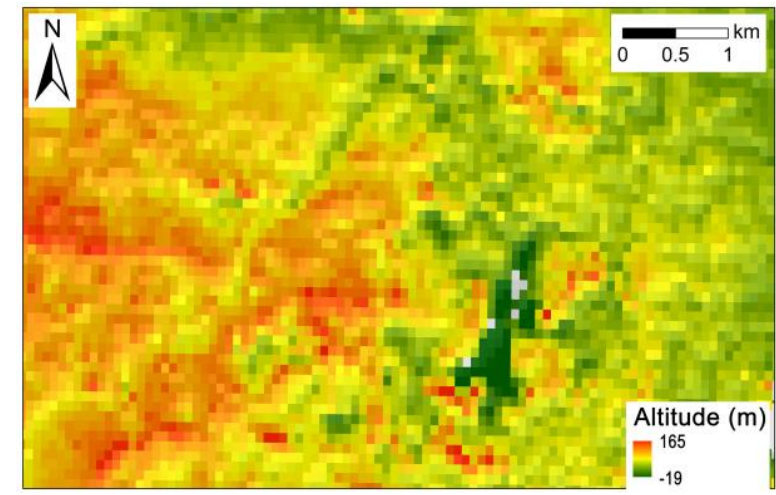

(c) SRTM version 2 DEM (90 m mesh).

Figure 5. Comparison among (a) AW3D DSM (5m mesh), (b) AW3D30 Ave DSM (30m mesh), and (c) SRTM version 2 (90m mesh) in a part of Tokyo, Japan.

slightly reduced as a demerit. Another is the medium method (Med), which is selected a medium height value i.e. $25^{\text {th }}$ height from 49 pixels. If it shows a masked value, same value is kept in AW3D30. This is likely keeping the terrain textures, however the fraction of masked values is not much changed. Actually, the nearest neighbour resampling method is the most suitable one to reduce pixel spacing, however it is sometime conflict with AW3D commercial bases distribution.

The both Ave and Med datasets are contained in individual AW3D30 dataset, which can be downloaded free of charge. Users can be considered to select one of them for own purposes. Figure 5 shows an example of visual image comparison among (a) AW3D DSM, (b) AW3D30 Ave DSM, and (c) SRTM ver.2 as just reference in a part of Tokyo, Japan. This is clearly identified the difference of the spacing, and AW3D30 DSM is still keeping terrain features. 


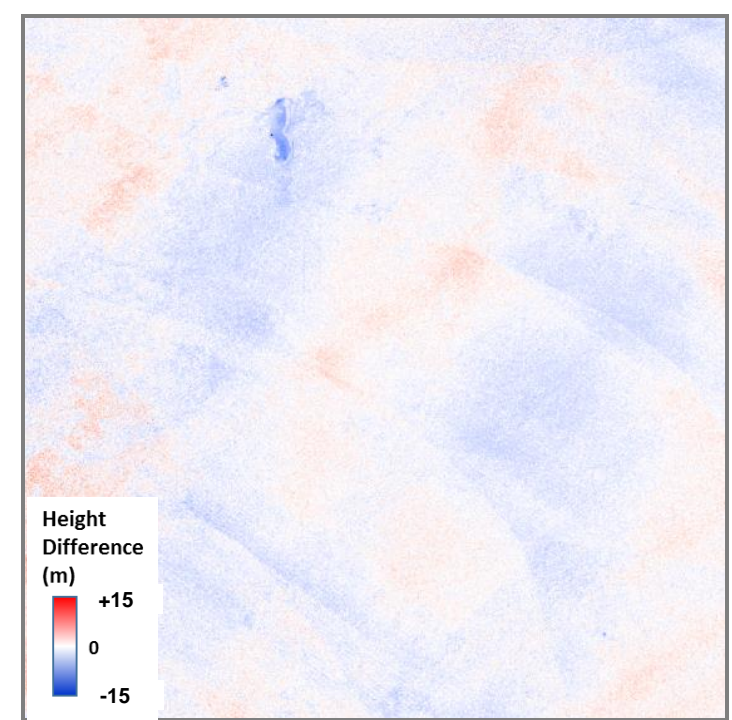

(a) S032E124.

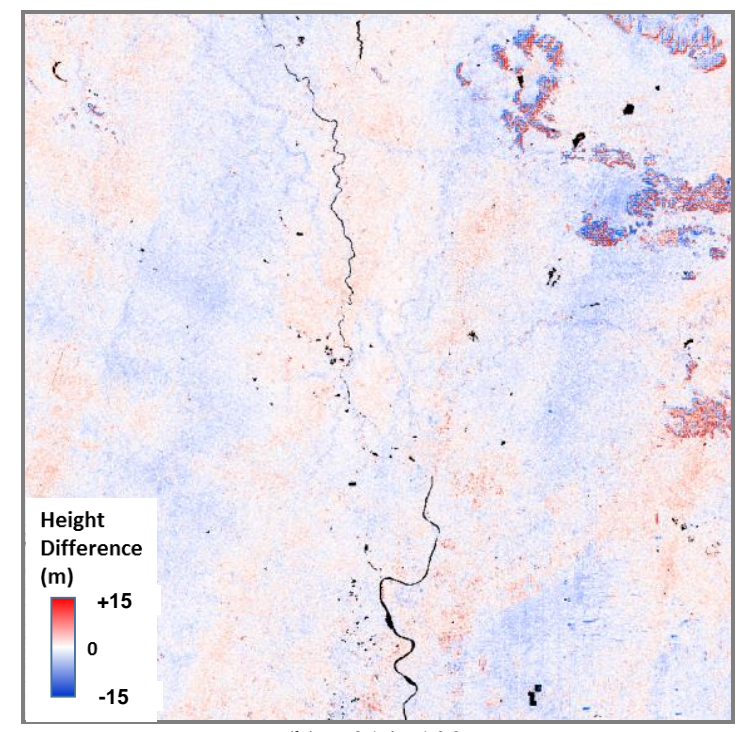

(b) N014E100.

Figure 6. Example of the height difference image between AW3D30 Ave DSM (30 m mesh) and SRTM-3 v.3 (90 m mesh).

\section{PRELIMINARY VALIDATION OF AW3D30 DSM}

The aim of AW3D30 dataset generation is to provide the global digital elevation data for research and science purposes but also commercial uses in fields of geo-spatial information and application. Although this dataset freely open to public, the height accuracy should be evaluated. Two validation studies are mainly conducted for AW3D30 DSM; 1) spatial consistency evaluation compared with existing DEM i.e. SRTM-3 version 3 (v.3) to identify systematic errors and masks status in widespread areas as a relative validation, and 2) an absolute validation using ground-based check points (CPs).

Figure 6 shows height difference images of AW3D30 DSM minus SRTM-3 v.3 for (a) tile no. S032E124 in southwest Australia, and (b) N014E100 in northern Bangkok, Thailand that an example of spatial consistency evaluations as relative validation, where are white colours indicate small height differences between them, red is AW3D30 higher than SRTM, blue is lower than that, and blacks are indicated masked areas due to remaining clouds and land water areas i.e. rivers and

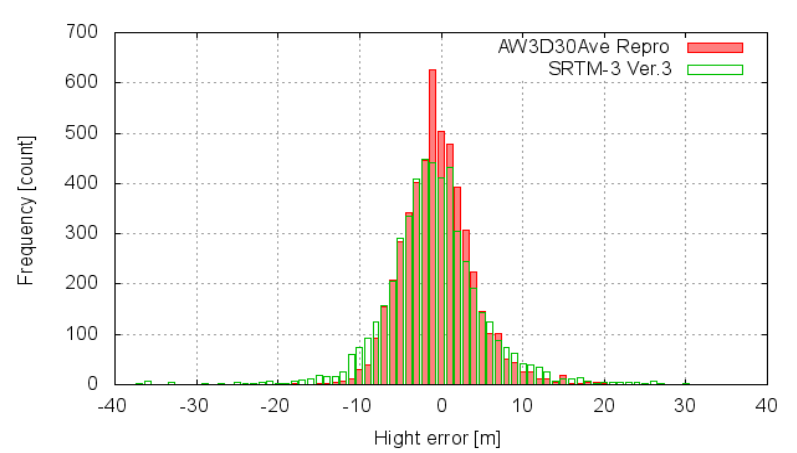

Figure 7. Histogram of height error evaluated by 5,121 CPs in 127 tiles (red: AW3D30 Ave DSM, and green: SRTM-3 version 3 as reference).

Table 3. Validation results by $5,121 \mathrm{CPs}$ in 127 tiles.

\begin{tabular}{|l|c|c|c|}
\hline Dataset & Ave. $(\mathrm{m})$ & STDEV $(\mathrm{m})$ & RMSE $(\mathrm{m})$ \\
\hline AW3D30 Ave & -0.44 & 4.38 & 4.40 \\
\hline Ref) SRTM v.3 & -1.00 & 7.43 & 7.50 \\
\hline
\end{tabular}

*Ave.: average, STDEV: standard deviation

lakes. There are some systematic height differences could be confirmed both (a) and (b). They look some gaps along with the SRTM's orbit in (a), and similar with the ALOS's orbit in (b). The height difference values are not so large except for some small patched areas in (b), which have relatively large height differences in northeast part. These may be actual differences due to land covers changes due to the observation time difference between SRTM (2000) and ALOS (2006 to 2011).

The absolute height validation of AW3D30 DSM datasets were conducted using 5,121 CPs distributed in 127 tiles that located in not only Japan area but also foreign countries. Figure 7 shows the histogram of height error evaluated by 5,121 CPs, where red bars are the result for AW3D30 Ave DSM, and green bars are for SRTM-3 v.3 as just reference. The shapes of histograms look Gaussian distribution with almost zero peaks, but AW3D30's one is much sharp than SRTM's one. Table 3 summarizes statics of evaluation of AW3D30 Ave DSM and SRTM-3 v.3 as just reference. It was confirmed $4.38 \mathrm{~m}$ (standard deviation, STDEV) and $4.40 \mathrm{~m}$ (RMSE) of height accuracy for AW3D30 Ave DSM, which was gained the target accuracy of $5 \mathrm{~m}$ (RMSE). Similar evaluations will be continued when new tiles are available.

\section{CONCLUSIONS}

This paper introduced generation and the preliminary validation result of new global digital elevation called the "ALOS World 3D 30 m mesh" (AW3D30) DSM with 1 arcsec. spacing, which is created based on AW3D $5 \mathrm{~m}$ mesh (0.15 arcsec.) DSM using archived ALOS PRISM stereo or triplet pair images. AW3D30 DSM dataset is open to the public free of charge, and can use for not only research communities but also commercial uses in fields of geo-spatial information and application.

As the preliminary validation result of AW3D30 DSM, $4.40 \mathrm{~m}$ (RMSE) height accuracy was confirmed from independent 5,121 CPs distributed in 127 tiles. However, it has sometimes small height gaps, which may causes in between orbit paths of ALOS. The remaining clouds are also problem to utilize application. We are considering to correct the height gaps in between paths, and interpolate the cloud areas by other sources i.e. SAR interferometry by ALOS-2 and the existing global DEMs in AW3D30 version 2. 


\section{ACKNOWLEDGEMENTS}

The authors thank our collaborators from NTT DATA Corp. and RESTEC for participating in the project, in particular, we are grateful to Mr. Junichi Takaku for his technical support and Dr. Ken Tsutsui for his management efforts.

\section{REFERENCES}

Farr, T. G., P. A. Rosen, E. Caro, R. Crippen, R. Duren, S. Hensley, M. Kobrick, M. Paller, E. Rodriguez, L. Roth, D. Seal, S. Shaffer, J. Shimada, J. Umland, M. Werner, M. Oskin, D. Burbank, and D. Alsdorf, 2007. The Shuttle Radar Topography Mission. Report on the Jet Propulsion Laboratory (JPL), California Institute of Technology, California, US

http://www2.jpl.nasa.gov/srtm/SRTM_paper.pdf (24 Apr. 2016).

Iwata, T., T. Tadono, T. Kawahara, and M. Abe, 2008. Precision Pixel Geolocation Determination for the Advanced Land Observing Satellite (ALOS). Proc. 59th International Astronautical Congress.

JAXA EORC, 2016. ALOS Global Digital Surface Model "ALOS World 3D - 30m" (AW3D30), http://www.eorc.jaxa.jp /ALOS/en/aw3d30/index.htm (24 Apr. 2016).

NASA JPL Online News, 2014.

http://www.jpl.nasa.gov/news/news.php?release=2014-321 (24 Apr. 2016).

Shimada, M., T. Tadono, and A. Rosenqvist, 2010. Advanced Land Observing Satellite (ALOS) and Monitoring Global Environmental Change. Proc. the IEEE, 98(5), pp. 780-799.

Tachikawa, T., M. Hato, M. Kaku, and A. Iwasaki, 2011. The characteristics of ASTER GDEM version 2. Proc. IGARSS 2011, IEEE, CD-ROM.

Tadono, T., M. Shimada, H. Murakami, and J. Takaku, 2009. Calibration of PRISM and AVNIR-2 onboard ALOS "Daichi". Transactions on Geoscience and Remote Sensing, IEEE, 47(12), pp. 4042-4050.

Tadono, T, T. Iwata, M. Shimada, J. Takaku, and S. Kawamoto, 2010. Updated results of calibration and validation of PRISM onboard ALOS. Proc. IGARSS 2010, IEEE, CD-ROM.

Takaku, J. and T. Tadono, 2009a. PRISM on-orbit geometric calibration and DSM performance. Transaction on Geoscience and Remote Sensing, IEEE, 47(12), pp. 4060-4073.

Takaku, J. and T. Tadono, 2009b. High resolution DSM generation from ALOS PRISM -Status updates on over three year operations-. Proc. IGARSS 2009, IEEE, CD-ROM.

Takaku, J. and T. Tadono, 2010. High resolution DSM generation from ALOS PRISM -Processing status and influence of attitude fluctuation-. Proc. IGARSS 2010, IEEE, CD-ROM.

Takaku, J. and T. Tadono, 2011. High resolution DSM generation from ALOS PRISM -Archiving and mosaicing-. Proc. IGARSS 2011, IEEE, CD-ROM.

Takaku, J., T. Tadono, K. Tsutsui, and M. Ichikawa, 2016. Validation of 'AW3D' Global DSM Generated from ALOS PRISM, Annals of the Photogrammetry, Remote Sensing and Spatial Information Sciences, ISPRS, submitted. 